\title{
Polarization of atomic radiation in stochastic plasma fields
}

\author{
V. I. Savchenko and N. J. Fisch \\ Princeton Plasma Physics Laboratory, Princeton University, Princeton, New Jersey 08543
}

(May 12, 1997)

\begin{abstract}
When a laser pulse of certain polarization or an electron beam excites atoms in a plasma, the atomic spectrum of the radiation emitted by the atoms exhibits differently polarized line core and line wings. This unusual effect, which is predicted to occur under a variety of conditions, can be accompanied by the appearance of the forbidden component in the spectrum, with polarization opposite to that of the exciting laser pulse.
\end{abstract}

PACS: $39.30,52.70,32.70 . J$

Radiation from atoms immersed in a plasma can be broadened through several means, including Doppler broadening, collisional broadening, and Stark broadening. The polarization of the broadened spectrum, however, need not be uniform over the line profile. This paper describes an interesting effect that accompanies atoms in a plasma that are prepared preferentially in one magnetic sublevel. The core and the wings of the radiation spectra from these atoms are polarized to different degrees.

The first such interesting polarization effects were discovered by E. K. Zavoiskii [1] and Sholin and Oks [2], where differences in the line core and wing degree of polarization in the optical range of frequencies arise from low-frequency turbulent electric fields in the plasma. Because there is a preferred direction to the plasma fields, the circularly polarized $\sigma$ components lie close to the center of the line, while the $\pi$ components split into the wings. Hence, the different polarizations exhibit different line profiles $[3,4]$. This effect also is reputedly responsible for the polarization of X-ray line profiles in Z-pinch plasma [5], even though the electric fields are now quasimonochromatic and intense enough to split up the line into satellites [6].

It is also true that an anisotropic electron velocity distribution can excite preferentially one magnetic sublevel $[7,8]$. Kieffer et al. measured polarized line emission in the presence of a beam of fast electrons [8]. Since the preferential excitation depends sensitively on beam directivity, moments of the electron velocity distribution could be deduced from differences in polarization.

We show, however, that if magnetic sublevels are preferentially excited in a plasma, there is not only the net polarization of the emission [8], but also differences in the degree of polarization between the line core and wings. The means of achieving the preferential excitation could be an electron beam [8] or polarized laser light.

First, suppose a distribution of microfields which is isotropic, but sharply peaked around a given amplitude. We find that emission in the line core is almost unpolarized while the line wings are almost completely polarized. This can be understood from the uncertainty principle:
Atoms radiating in a time $\Delta \tau$ emit into the line wings at a characteristic frequency detuning $\Omega \equiv \omega-\omega_{0} \sim 1 / \Delta \tau$, where $\omega_{0}$ is the resonant frequency. Let $T$ be the characteristic time for mixing of the magnetic sublevels in the presence of the plasma microfields, which we assume to be less than the decay time. For $\Delta \tau \ll T$, an atom does not have time to change its state before it decays; hence, it emits a photon of polarization corresponding to the preferentially excited sublevel. On the other hand, for $\Delta \tau \geq T$, the atomic states are mixed due to plasma microfields prior to the line emission. Thus, emission into the wings, arising from short radiation times, should be polarized whereas, emission into the core, arising from long radiation times should be much less polarized. Of course, in a plasma, if the distribution of the microfields is not sharply peaked around a given amplitude, then the observed radiation would be an averaged effect over a distribution of amplitudes. As shown below, this average can retain important features of the sharply peaked case.

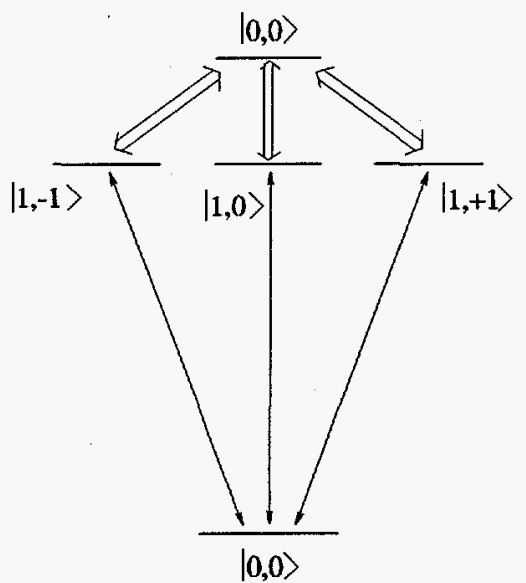

FIG. 1. Double lines represent mixing of levels due to static electric field; thin lines correspond to spontaneous decay of $\sigma$ - levels. Upper levels are degenerate.

Incidentally, this effect should occur also in the presence of the polarization effects discovered in the Z-pinch experiment reported by Oks [5], because the axial cur- 


\section{DISCLAIMER}

This report was prepared as an account of work sponsored by an agency of the United States Government. Neither the United States Government dor any agency thereof, nor any of theis employeer, makes any warnaty, express or implied, or sesumes any legal liability or responsibility for the accuracy, completeness, or usefulness of any information, apparatus, produce, or process disclosed or represents that its use would not infringe privately owned rights. Reference berein to any specific commercial product, process, or service by trade name, trademarit, manufacturet. or otherwise does not necessarily constitute or imply its endorsemeat, recommendation. or favoring by the United States Government or any agreacy thereof. The views and opinions of authors expressed berein do aot necossanly sate or reflect those of the. United States Government or any agency thereof. 


\section{DISCLAIMER}

Portions of this document may be illegible in electronic image products. Images are produced from the best available original document. 
rent should preferentially excite $\pi$ states. Thus, the differences in degree of polarization between the core and wings could be attributed both to the turbulent fields and the differences in the initial populations.

In order to find the line profile in a plasma, it is necessary to take into account the coherency between atomic sublevels, something that is accomplished using the density matrix approach [9].

In order to gain insight into spontaneous emission of an atom in stochastic plasma fields, consider first the case of a constant electric field, where an atom is excited into one magnetic sublevel. Suppose the atomic structure depicted in Fig. 1. Transitions occur between the degenerate upper level $\mid J=1, m=0, \pm 1>$ and the lower level $\mid J=0, m_{j}=0>$. The external electric field couples the upper level $\mid J=0, m=0>$ to the $\sigma=\mid J=1, m=0, \pm 1>$ levels. Let the $z$-axis be along the direction of a circularly polarized laser pulse. (The case of linear polarization is handled similarly.)

Projecting the Schrodinger equation onto eigenfunctions of the angular momentum, we get a system of linear differential equations for the amplitudes

$$
i \frac{d a_{\sigma}}{d t}=V_{\sigma u} a_{u}, \quad i \frac{d a_{u}}{d t}=\sum_{\sigma} V_{u \sigma} a_{\sigma}
$$

where $V_{u \sigma}=V E_{\sigma}^{*}=d_{u \sigma} E E_{\sigma}^{*}$ is a matrix element of the interaction with electric field, and $E$ and $E_{\sigma}$ are the amplitude and dimensionless spherical component of the electric field respectively.

For initial conditions $a_{\sigma}(t=0)=\delta_{\sigma \alpha}$, Eqs. (1) have the solution

$$
\begin{gathered}
a_{\sigma}=E_{\alpha}^{*} E_{\sigma}(\cos V t-1)+\delta_{\sigma \alpha} \\
a_{u}=-i E_{\alpha}^{*} \sin V t,
\end{gathered}
$$

where $\alpha$ stands for the polarization of the pulse.

For a right-hand circularly polarized laser pulse $\alpha=1$, Eqs. (2) and (3) give the expectation value of the dipole moment of the transition between upper and lower levels as

$$
\begin{aligned}
\mathbf{d}= & \sqrt{2} d\left[\left(E_{x}^{2} \mathbf{e}_{x}+E_{x} E_{z} \mathbf{e}_{z}\right)(1-\cos V t) \cos \omega_{0} t-\right. \\
& \left.\left(\mathbf{e}_{x} \cos \omega_{0} t+\mathbf{e}_{y} \sin \omega_{0} t\right)\right]
\end{aligned}
$$

where the electric field is assumed to lie in the $x-z$ plane. Note that the tip of the dipole moment vector describes an elliptical path in time $1 / \omega_{0}$; the plane of this path, initially in the $x-y$ plane, oscillates around $y$-axis with frequency $1 / V$. The electric field behaves similarly, as shown in Fig. 2.

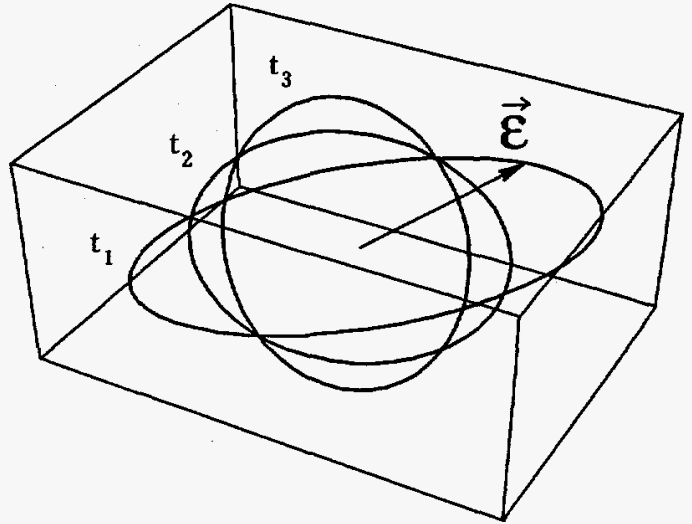

FIG. 2. Polarization of radiation when $E_{z} \neq 0$.

Consider now a laser pulse say of right-hand circular polarization, exciting an atom that is subjected to the stochastic microfields of a plasma. The line formation can be treated in two limits, impact and quasi-static [10], corresponding to emission into the line core and line wings. Frequency detuning $\Omega$ less than the Weisskopf frequency $\omega_{W}[11]$ corresponds to the line core, while detuning greater than $\omega_{W}$ corresponds to the line wings.

The atomic spontaneous emission spectrum can be written as [12]

$$
R(\omega)=2 h \omega R e\left[i G_{\sigma} \rho_{g \sigma}(s=-\Omega)\right],
$$

where $G_{\sigma}$ is the matrix element of the Hamiltonian of the interaction between an atom and the spontaneous field, with polarization $\sigma$ and frequency $\omega$, and where $\rho_{g \sigma}(s=-\Omega)$ is the Laplace transformed atom + field density matrix element, $\rho_{g \sigma}(t)$, evaluated at $s=-\Omega$.

Assume the spontaneous field is sufficiently weak that only transitions from the upper level to the lower one are induced. Hence, the equations for the atom + spontaneous field density matrix, in the quasistatic limit, can be written as $[9,13]$ :

$$
\begin{aligned}
& i \dot{\bar{\rho}}_{g \sigma}=-\bar{\rho}_{g u} V_{u \sigma}+(\Omega-i \Gamma) \bar{\rho}_{g \sigma}+\sum_{\sigma^{\prime}} G_{\sigma^{\prime}}^{*} \rho_{\sigma^{\prime} \sigma}^{0} \\
& i \dot{\bar{\rho}}_{g u}=-\sum_{\sigma} \bar{\rho}_{g \sigma} V_{\sigma u}+\Omega \bar{\rho}_{g u}+\sum_{\sigma^{\prime}} G_{\sigma^{\prime}}^{*} \rho_{\sigma^{\prime} u}^{0},
\end{aligned}
$$

where $\rho(t)=\bar{\rho}(t) e^{i \Omega t}, \rho_{\sigma \sigma^{\prime}}^{0}$ is the atomic density matrix, and where $\Gamma$ is the radiation decay constant.

For simplicity, we neglect collisional contributions to the line broadening. This can be justified, i.e., in a turbulent plasma, when the influence of wave fields, $E_{f}$, becomes dominant over fields, $E_{p}$, due to single particles, $\left\langle E_{f}^{2}\right\rangle^{1 / 2} / \bar{E}_{p} \gg 1$. It is known that the effect of these fields on radiating atoms can be described in the quasistatic approximation [11]. In thermal plasmas, this procedure is valid, when electrons are quasistatic [11], or when electron collisional contribution, $\nu_{e}$, is smaller than the radiative width, $\Gamma$, which scales as $Z^{4}$ for multiply 
charged ions. Note, that $\Gamma$ has to be of the order of quasistatic ion width, $w_{i}$, to ensure quasistatic $m$-mixing, essential for the effects described in this paper. These requirements lead to the constraint on the ion density, $N_{i} \ll 10^{14} Z^{5} T_{e}^{1 / 2}(\mathrm{eV}), N_{i} \sim 0.5 \cdot 10^{15} Z^{4} \mathrm{~cm}^{-3}$, which can be satisfied for $Z \sim 10$ and $T_{e}>1 \mathrm{eV}$.

Also, the contributions of the ion motion of the ambient plasma to the line boadening can be neglected in most cases of interest [11], which means that the ion fields can be viewed as quasistatic. If Stark broadening is larger than Doppler broadening, the radiating ions can be assumed to be stationary; otherwise, the theory presented here is easily generalized.

The inverse matrix for the inhomogeneous system of equations (6) and (7) is

$$
\begin{gathered}
\left(D^{-1}\right)_{\sigma \sigma^{\prime}}=\frac{\Omega-i \Gamma}{\operatorname{det} D}\left(\left[V^{2}-\Omega^{2}+i \Omega \Gamma\right] \delta_{\sigma \sigma^{\prime}}-V_{\sigma}^{*} V_{\sigma^{\prime}}\right) \\
\left(D^{-1}\right)_{\sigma u}=-\frac{\Omega-i \Gamma}{\operatorname{det} D} V_{\sigma}^{*} \\
\operatorname{det} D=(\Omega-i \Gamma)^{2}\left[\Omega(\Omega-i \Gamma)-V^{2}\right] .
\end{gathered}
$$

The atom + field density matrix element, $\left.\bar{\rho}_{g \sigma}\right|_{s=0}$, which enters Eq. (5), can now be written as

$$
\left.\rho_{g \sigma}\right|_{s=0}=\sum_{\sigma^{\prime} \nu} D_{\sigma \nu}^{-1} \rho_{\sigma^{\prime} \nu}^{0} G_{\sigma^{\prime}}^{*}
$$

It remains, of course, to find the $\rho_{\sigma \sigma^{\prime}}^{0}$.

The atomic density matrix equations can be written as,

$$
\begin{aligned}
& i \dot{\rho}_{\sigma^{\prime} \sigma}^{0}=V_{\sigma^{\prime} u} \rho_{u \sigma}^{0}-\rho_{\sigma^{\prime} u}^{0} V_{u \sigma}-i 2 \Gamma \rho_{\sigma^{\prime} \sigma}^{0} \\
& i \dot{\rho}_{\sigma u}^{0}=V_{\sigma u} \rho_{u u}^{0}-\sum_{\sigma^{\prime}} \rho_{\sigma \sigma^{\prime}}^{0} V_{\sigma^{\prime} u}-i \Gamma \rho_{\sigma u}^{0} \\
& i \dot{\rho}_{u u}^{0}=\sum_{\sigma}\left(V_{u \sigma} \rho_{\sigma u}^{0}-\rho_{u \sigma}^{0} V_{\sigma u}\right)
\end{aligned}
$$

which form a homogeneous system of equations of the form $i \dot{\vec{\rho}}=\mathbf{M} \cdot \vec{\rho}$, where $\mathbf{M}$ is a $16 \times 16$ matrix, to be solved with initial conditions $\rho_{\nu \nu^{\prime}}^{0}(t=0)=\delta_{\alpha \alpha}$, where $\nu$ stands for both $\sigma$ and $u$, and $\alpha$ is the polarization of the laser pulse which excited the atom at time $t=0$.

The standard technique for solving Eqs. (12) - (14) is to Laplace transform and then invert the fundamental matrix, which is large. However, a much simpler approach can be employed in the regime we consider, which in the end enables us to find a simple analytic form for the polarization. Thus, we can write

$$
\left([M-I s]^{-1}\right)_{\nu \nu^{\prime}}^{\nu_{1} \nu_{2}}=\int_{0}^{\infty} e^{i s t}\left[A_{\nu \nu_{1}}(t) A_{\nu^{\prime} \nu_{2}}^{*}(t)\right] d t
$$

where $A_{\nu \nu_{1}}(t)$ is an evolution matrix for the amplitudes found from the system of Eqs. (1), modified to include damping terms $\Gamma a_{\sigma}$. The $A_{\nu \nu_{1}}(t)$ can be calculated by inverting only a $4 \times 4$ matrix, and upon substitution into Eq. (15), we get

$$
\begin{gathered}
\rho_{\sigma \sigma^{\prime}}^{0}=\left|E_{\alpha}\right|^{2} E_{\sigma} E_{\sigma^{\prime}}^{*} 2 g-\left(E_{\sigma^{\prime}}^{*} E_{\alpha} \delta_{\sigma \alpha}+E_{\sigma} E_{\alpha}^{*} \delta_{\sigma^{\prime} \alpha}\right) g \\
+\frac{1}{2 \Gamma} \delta_{\sigma \alpha} \delta_{\sigma^{\prime} \alpha} \\
\rho_{\sigma u}^{0}=\left(-\left|E_{\alpha}\right|^{2} E_{\sigma}+E_{\alpha} \delta_{\sigma \alpha}\right) \frac{i V}{V^{2}+2 \Gamma^{2}} \\
g=\left(\frac{1}{2 \Gamma}-\frac{\Gamma}{V^{2}+2 \Gamma^{2}}\right)
\end{gathered}
$$

Thus, using $\rho_{\nu \nu}^{0}$, from Eqs. (16) - (18) in Eq. (11), we have

$$
\begin{aligned}
I_{\sigma \sigma^{\prime}} \propto & \left|\frac{\Omega-i \Gamma}{\operatorname{det} D}\right|^{2}\left(\frac{1}{15} V^{4} \delta_{\sigma \sigma^{\prime}}+\right. \\
& \left.\frac{1}{6}\left[3 \Omega^{4}+V^{4}-\Omega^{2} V^{2} \frac{4 V^{2}+5 \Gamma^{2}}{V^{2}+2 \Gamma^{2}}\right] \delta_{\sigma \alpha^{\prime}} \delta_{\sigma^{\prime} \alpha}\right) .
\end{aligned}
$$

The degree of a circular polarization can now be calculated as,

$$
P^{w}(\Omega)=\frac{\gamma}{1+\gamma}, \quad \gamma=\frac{1}{2}\left(\frac{I_{+}}{I_{-}}-1\right)
$$

$$
\gamma=\frac{5}{4} \frac{1}{V^{4}}\left[3 \Omega^{4}+V^{4}-\Omega^{2} V^{2} \frac{4 V^{2}+5 \Gamma^{2}}{V^{2}+2 \Gamma^{2}}\right]
$$

for initial conditions $\rho_{\nu \nu^{\prime}}^{0}(t=0)=\delta_{11}$. The simple analytic form for the polarization, Eq. (20), is a principal result of this work. In Fig. 3, we show $P^{w}(\Omega)$ appropriately averaged over different microfield distributions.

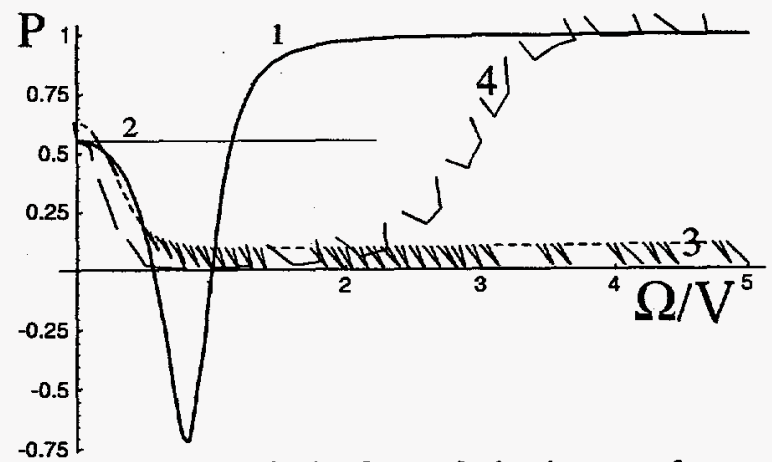

FIG. 3. Degree of circular polarization vs. frequency: curves 1 (quasistatic) and 2 (impact) correspond to $P(\Omega)$ at one particular value of the microfield, $V$, with $\Gamma / V=0.01$; For curves 3 and $4, V$ represents an averaged quantity over the corresponding microfield distribution, Holtzmark or Gaussian, plotted for the case $\Gamma / V=0.01$. 
In the impact limit, $\Omega<\omega_{W}$, the equations for the atom + field density matrix can be put into the irreducible form [14]

$$
i \dot{\bar{\rho}}_{q}^{k}=\left[\Omega-i \bar{\Gamma}_{k}\right] \bar{\rho}_{q}^{k}+\sum_{\sigma \sigma^{\prime}}(-1)^{\sigma} C_{001-\sigma}^{k q} G_{\sigma^{\prime}}^{*} \rho_{\sigma^{\prime} \sigma}^{0}
$$

where $k$ and $q$ stand respectively for the amplitude and projection of the angular momentum resulting from addition of angular momenta of the lower and upper levels, $C_{001-\sigma}^{k q}$ is a Clebsh-Gordon coefficient, and $\bar{\Gamma}_{k}=\Gamma+g_{k}$, with $g_{k}$ being a rate of destruction of coherency between upper and lower levels. The inverse of the fundamental matrix in $\sigma$ representation is

$$
\left(H^{-1}\right)_{\sigma \sigma^{\prime}}=\frac{1}{i \bar{\Gamma}_{1}-\Omega} \delta_{\sigma \sigma^{\prime}}
$$

Making use of Eqs. (5), (16) - (18), (22), (23) we can write down the polarization matrix of the emitted photon

$$
I_{\sigma \sigma^{\prime}} \propto \frac{\bar{\Gamma}_{1}}{\Gamma} \frac{\left(\frac{2}{15} V^{2} \delta_{\sigma \sigma^{\prime}}+\frac{1}{3}\left(V^{2}+3 \Gamma^{2}\right) \delta_{\sigma \alpha} \delta_{\sigma^{\prime} \alpha}\right)}{\left(V^{2}+\Gamma^{2}\right)\left(\Omega^{2}+\bar{\Gamma}_{1}^{2}\right)}
$$

which is valid for the line core. Thus, from Eqs. (20) and (24), the degree of the circular polarization is

$$
P^{c}(\Omega)=\frac{5}{9} \frac{1+3(\Gamma / V)^{2}}{1+5 / 3(\Gamma / V)^{2}},
$$

which is independent of the frequency detuning, $\Omega$ (line 2 in Fig. 3). Note from Fig. 3 (line 1) that the line wings are almost completely polarized, while the line core is only partially polarized, in agreement with the qualitative predictions outlined in the introduction. Note also a prominent dip in curve 1 , which means that, at the frequency $\Omega \sim V$, the emitted photon is predominantly counterpolarized, namely, left-hand polarized. The fact that $I_{-}$is greater than $I_{+}$at the frequency detuning $\Omega \sim V$ arises from Stark oscillations of $\rho_{g \sigma}$ at frequency $V$, with initial conditions $\rho_{\sigma \sigma^{\prime}}(t=0)=\delta_{\sigma 1} \delta_{\sigma^{\prime} 1}$.

Note that Eqs. (21) and (25) refer to the case of microfields of fixed amplitude, whereas, in a plasma, there is a distribution of amplitudes depending on, among other things, the number of particles in a Debye sphere. Corresponding to the limits of infinite and zero number of particles in the Debye sphere are the Holzmark and nearestneighbor approximations, respectively. Averaging with respect to a Holtzmark [11] field distribution we obtain curve 3 in Fig. 3; an averaging with respect to a nearestneighbour ion distribution yields a very similar result. Thus, the degree of polarization is fairly insensitive to the plasma parameter regime. Note that, upon averaging, both the counterpolarized feature and the polarization in the wings become muted. For the case of static ionic broadening, polarization remains only in the line core. However, in principle, nonequilibrium distributions could exhibit a peaked distribution of amplitudes, which would then result in counterpolarized forbidden component in the broadened spectrum. The fact that different microfield distributions lead to different polarization features could inform importantly on the plasma state.

Alternatively, consider a Gaussian distribution of fluctuating fields, generated by waves in an isotropic plasma at thermodynamic equillibrium. Curve 4 in Fig. 3 represents an averaging over such fields. Note that such averaging retains both polarization in the wings and a pronounced minimum. This striking difference between the influence of particle and wave fields on the degree of polarization could serve to distinguish between the relative intensities of long wavelength and short wavelength stochastic fields.

In summary, in the simplest configuration of levels that exhibits polarization effects, we have found that the degree of polarization over the line profile of emission from atoms prepared in one magnetic sublevel depends upon the plasma microfield distribution. For the case of a Gaussian distribution, which describes turbulent wave fields, we find complete polarization in the wings.

The authors are grateful to Dr. A. A. Panteleev for useful comments. This work was supported by the United States Department of Energy under Contract No. DEAC02-CHO-3073.

[1] E. K. Zavoiskii et al., ZhETF Pis. Red. 13, 19 (1971).

[2] G. V. Sholin and E. A. Oks, Sov. Phys. Dokl. 18, 254 (1973).

[3] M. V. Babykin et al., Sov. Phys. JETP 38, 86 (1974).

[4] C. Deutch and G. Bekefi, Phys. Rev. A 14, 854 (1976).

[5] E. A. Oks, in Proceedings on the 13-th International Conference on Spectral Line Shapes (AIP, New York, 1997), p. 11.

[6] E. A. Oks, Plasma Spectroscopy (Springer-Verlag, New York, 1995).

[7] J. C. Kieffer et al., Phys. Rev. Lett. 68, 480 (1992).

[8] J. C. Kieffer et al., Phys. Rev. E 48, 4648 (1993).

[9] M. Sargent, M. O. Scully, and W. E. Lamb, Laser Phisics (Addison-Wesley, London, 1974).

[10] I. I. Sobelman, Introduction to the Theory of Atomic Spectra (Fizmatgiz, Moscow, 1963).

[11] H. R. Griem, Spectral Line Broadening by Plasmas (Academic Press, New York, 1974).

[12] S. G. Rautian, G. I. Smirnov, and A. M. Shalagin, Nonlinear Resonances in Atomic and Molecular Spectra (Nauka, Moscow, 1979), in Russian.

[13] A. N. Starostin et al., Zh. Eksp. Teor. Fiz. 98, 1304 (1990).

[14] J. Cooper, R. J. Ballagh, and K. Burnett, Phys. Rev. A 22, 535 (1980). 